\title{
Technik, Taktik und Grenzen der schweren, kombinierten Handverletzung
}

\author{
Amir Farhang Gharagozlou, Adrien Daigeler, Marcus Lehnhardt, Andrej Ring
}

\section{Zusammenfassung}

Komplexe Handverletzungen stellen meist traumatisch bedingte, multistrukturelle Verletzungen dar, welche umfangreiche Anforderungen an die operative Therapie sowie postoperative Behandlung stellen. Hierbei ist neben einer korrekten Indikationsstellung als Grundlage zur Planung der Operationsstrategie eine anschließende umfangreiche Rehabilitation notwendig, um eine bestmögliche Rekonvaleszenz zu gewährleisten. Daher empfiehlt sich die Behandlung in spezialisierten Zentren mit entsprechenden personellen und materiellen Voraussetzungen.

\section{Techniques, Strategies and \\ Limitations for Severe Combined Hand Injuries}

Severe combined hand injuries are mostly traumatically induced with multi-structural damage. They need a comprehensive operative treatment and also an intensive postoperative treatment. Apart from a proper indication as a basis for planning the surgical strategy a subsequent extensive rehabilitation is necessary to ensure the best possible recovery. Therefore, treatment in specialised centres with appropriate manpower and material conditions is recommended.
Unter einer komplexen Handverletzung versteht man die gleichzeitige Verletzung mehrerer anatomischer und funktioneller Strukturen mit der Gefahr eines Funktions- und/oder Sensibilitätsverlusts oder dem Verlust des betroffenen Körperteils.

Dementsprechend ist die Qualität der Behandlung komplexer Handverletzungen von besonderer Bedeutung, da dadurch die weitere Einsatzfähigkeit der gesamten oberen Extremität im Alltag und Beruf und damit die Erwerbsfähigkeit und Lebensqualität des Patienten beeinflusst wird.

Ursächlich für komplexe Handverletzungen sind i.d.R. Unfälle mit großer Energieeinwirkung (z.B. Quetschung, Avulsion, Explosion, Kreissäge).
OP-JOURNAL 2015; 31: 131-135

(c) Georg Thieme Verlag KG Stuttgart · New York DOI http://dx.doi.org/10.1055/s-0035-1546264
Das Behandlungsergebnis einer komplexen Handverletzung wird außer durch das Verletzungsmuster in nicht unwesentlichem Maße durch eine adäquate Erstbehandlung mit korrekter Indikationsstellung und suffizienter Nachbehandlung mit dem Ziel einer frühfunktionellen Therapie bestimmt.

Die unterschiedlichen funktionellen Strukturen, welche bei komplexen Handverletzungen beteiligt sein können, sind: der Weichteilmantel, die Muskulatur, die Gefäße, die Sehnen und Nerven sowie der Knochen. Einen Großteil komplexer Handverletzungen stellen die totalen bzw. subtotalen Amputationsverletzungen dar.

Komplexe Handverletzungen sollten, wenn immer möglich, in dafür geeigneten Zentren mit entsprechenden personellen und infrastrukturellen Voraussetzungen behandelt werden.

Das Operationsteam sollte fundierte Kenntnisse der Handchirurgie besitzen und zudem Erfahrung im Bereich der Mikrochirurgie und der Wiederherstellung von Weichteilen mittels Nahlappen, gestielten Fernlappen und freien Lappentransplantaten. In dieser Einrichtung müssen auch alle notwendigen sekundären Eingriffe durchführbar sowie im idealen Fall eine adäquate frühfunktionelle physio- und ergotherapeutische Behandlung möglich sein.

Der überwiegende Teil komplexer Handverletzungen ist traumatisch bedingt $(\mathrm{Ar}$ beitsunfälle, Verkehrsunfälle, landwirtschaftliche Unfälle, private Unfälle). $\mathrm{Zu}$ den Ursachen der Verletzungen zählen häufig holzbe- und -verarbeitende Maschinen (Kreissägen, Kettensägen, Holzspaltmaschinen). Leider kommt es auch immer noch zu schweren Ausrissverletzungen infolge unzureichender oder falsch verstandener Arbeitsschutzmaßnahmen. So sind z. B. Daumen- oder Fingerausrissverletzungen häufig durch das Tragen von Arbeitshandschuhen bei der Bedienung von Maschinen mit rotierenden Werkzeugen bedingt (Bohrmaschinen, Drehmaschinen, Fräsmaschinen).

Die optimale Planung und Versorgung komplexer Handverletzungen beinhaltet nicht nur den möglichen Erhalt der betroffenen Region, sondern sollte auch immer eine best- und schnellstmögliche Wiederherstellung der Funktion der gesamten Hand, angepasst an die beruflichen und sozialen Anforderungen des Einzelnen, berücksichtigen. Hierbei sollten wichtige Fragen im Vorfeld geklärt werden. Besteht eine Rekonstrukionsfähigkeit und ist der Patient für eine Rekonstruktion geeignet?

Die Indikation zur Rekonstruktion sollte immer vom verantwortlichen Operationsteam im Zentrum gestellt werden.

Viele Patienten glauben in der heutigen Wunschmedizin, dass das Wiederannähen des Amputats zu einer normalen, schmerzfreien Funktion führen wird und nahezu alle Patienten mit komplexen Handverletzungen sind mit dem auf- 


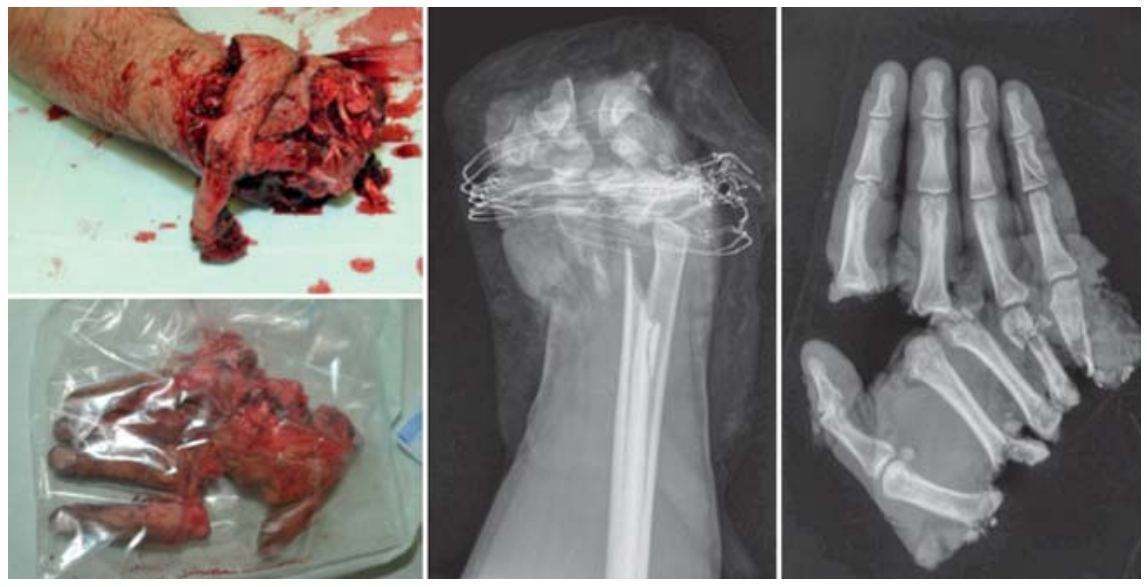

Abb. 1 33-jähriger Patient mit komplexer Quetschverletzung der rechten Hand. Trotz korrekter Amputatasservation war keine Rekonstruktion mit Replantation möglich. Bei der nativradiologischen Aufnahme zeigte sich eine völlige Destruktion der Radiuskonsole, eine Zerreißung aller ligamentären Strukturen der Handwurzelreihe bei Mehretagenverletzung des Amputats.
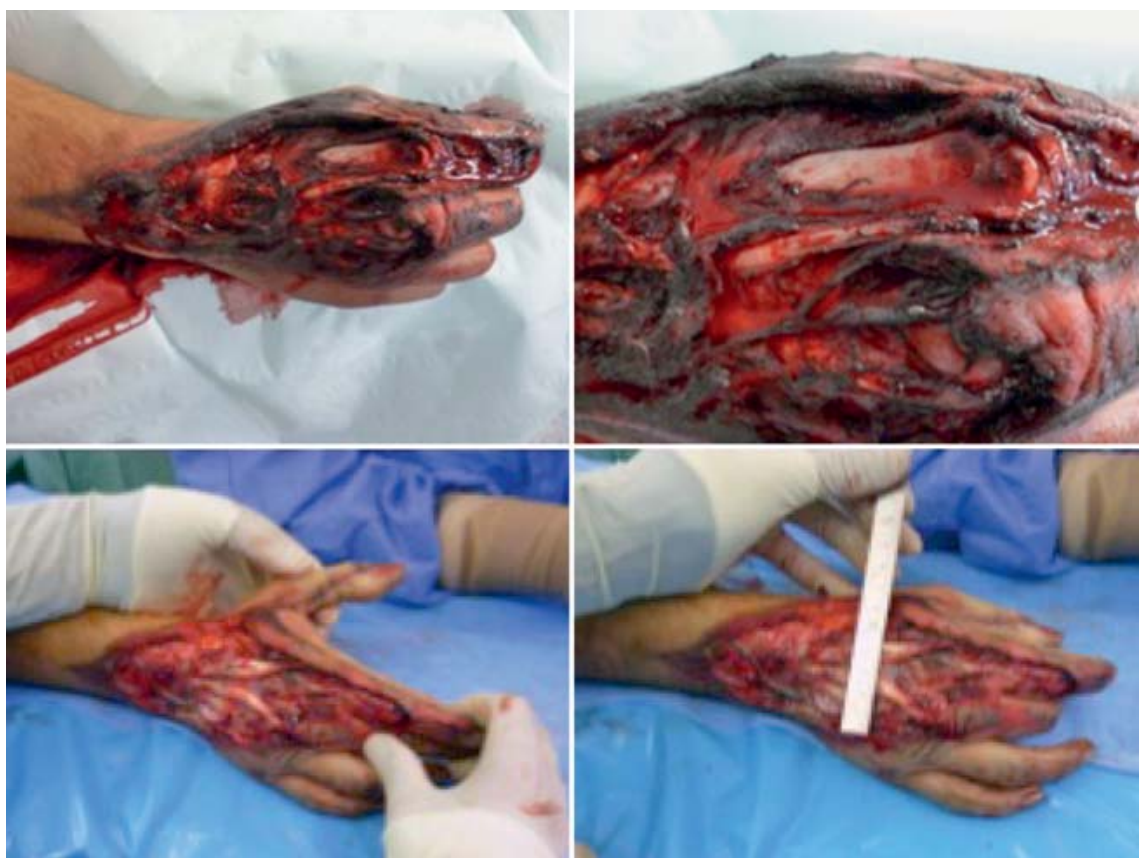

Abb.2a 29-jähriger Patient, welcher mit der rechten Hand in eine Hochgeschwindigkeitspoliermaschine geraten ist. Trotz Tragen eines Schutzhandschuhs kam es zu einem großen Haut-Weichteil-Defekt am Handrücken mit großflächiger Einsprengung von Farb- und Schmutzpartikeln, langstreckigem Strecksehnendefekt und einer Gelenkkapseldestruktion. Initial erfolgte ein radikales Débridement mit Resektion der betroffenen Haut-/Weichteile unter Schonung der Sehnen und Gefäße, um eine zweizeitige Rekonstruktion zu ermöglichen. Schon in der notfallmäßigen Versorgung wurden die Defektmaße erhoben und die Gefäße markiert, um die Rekonstruktion im Verlauf korrekt planen und durchführen zu können. Es erfolgte ein temporärer Weichteilverschluss mit Epigard, eine Schienenruhigstellung sowie eine intravenöse Antibiose.

wendigen Eingriff einverstanden oder fordern diesen sogar. Operationsart und -häufigkeit mit langen Krankenhausaufenthalten, multiple Folgeoperationen mit postoperativen Schmerzen, ein hohes Maß an Eigenverantwortlichkeit und -initiative in der Nachbehandlung und der mögliche Verlust des Arbeitsplatzes aufgrund langer Krankheitsdauer sind dem akut verletzten Patienten häufig nicht bewusst. Deshalb sollte eine schonende, jedoch richtungsweisende, adäquate Aufklärung des Patienten durchgeführt und dokumentiert werden (Abb. 1).Zudem sind Begleitverletzungen anderer Körperregionen auszuschließen, um das Risiko des Auftretens von Mono-, Oligo- oder Multiorganversagen und das Risiko des Versterbens nach langer Operationsdauer zu minimieren.
Das am Unfallort durch den Notarzt erhobene Verletzungsmuster der Hand spiegelt das wirkliche Verletzungsausmaß meist nur unzureichend wieder, weshalb eine genaue Untersuchung der Verletzungen am besten durch den Operateur erfolgen sollte.

Hierdurch können entsprechende Anforderungen an den Operationssaal bez. Lagerung, notwendiger Ausstattung (Mikroskop, bestimmtes Instrumentarium) sowie der zu erwartende Eingriff und die OP-Zeit festgelegt werden. Im Vorfeld sollte bei telefonischer Ankündigung einer komplexen Handverletzung mit dem OP-Manager eruiert werden, ob eine zeitgerechte Operation des Verletzten beim aktuellen Operationsaufkommen realisierbar ist. Hierbei gilt es, stundenlange Wartezeiten und damit eine Erhöhung der Ischämiezeit und des Infektrisikos zu vermeiden und den Patienten ggf. frühzeitig in ein anderes Zentrum zu verlegen.

Nach dem Eintreffen ist eine sofortige, sorgfältige Untersuchung des gesamten Patienten mit Ausschluss zusätzlicher, möglicherweise vital bedrohlicher Verletzungen durch den Operateur durchzuführen.

Hierbei ist die Anamnese mit Erfassung relevanter zusätzlicher Erkrankungen, Medikamenteneinnahme, Alter, Beruf, Freizeitinteressen, Allergien und anderer Faktoren, wie z.B. Nikotinkonsum, von besonderer Bedeutung.

Zudem sollte unbedingt die Sensibilitätsprüfung vor einer evtl. geplanten Narkose durchgeführt werden.

Immer ist auch der genaue Unfallhergang und -mechanismus zu eruieren, da die weitere Planung maßgeblich hierdurch beeinflusst wird. Zum Beispiel sollten bei Quetschtraumata eher zweizeitige Rekonstruktionen durchgeführt werden, da das Ausmaß des Gewebstraumas die weitere Therapie und Prognose wesentlich beeinflussen kann und bei Aufnahme noch nicht absehbar ist (Abb. 2a und b).

Neben der körperlichen Untersuchung zählt das konventionelle Röntgen weiterhin als wichtiges diagnostisches Mittel.

Erst bei unklarem Befund sollte eine weitere CT-Diagnostik erfolgen. Nach Festlegung einer OP-Strategie (Rückzugsmöglichkeiten, Varianten) muss 

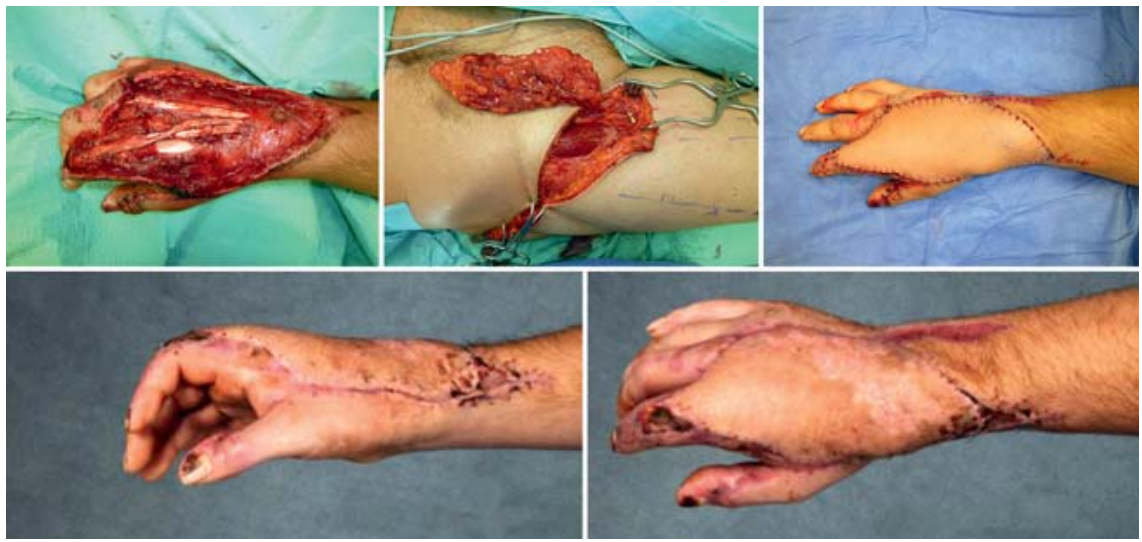

Abb. 2b Im 2. Schritt erfolgte nach Demarkierung des Haut-/Weichteildefekts und Ausschluss einer Infektbildung nach 5 Tagen die lappenplastische Deckung mittels freien Leistenlappen sowie die Rekonstruktion des Strecksehnenapparats mit Sehneninterposition. Bei Entlassung zeigte sich der Faustschluss fast komplett möglich bei gutem funktionellem Ergebnis.

eine kurze, zielorientierte Aufklärung des Patienten erfolgen.

Ziel ist ein rascher Beginn der Operation.

Diese beinhaltet maßgeblich ein radikales Débridement nicht erhaltungswürdiger Strukturen (v.a. Muskulatur). Nach Möglichkeit sollten kritische Strukturen wie Nerven, Sehnen und Arterien sowie gut erhaltene und vaskularisierte Gewebeanteile, auch wenn diese keine natürliche Funktion mehr haben, bis zum Schluss geschont werden, um für den Gebrauch bei der Rekonstruktion oder zur Weichteilbedeckung (Filetlappen, Hauttransplantate) benutzt werden $\mathrm{zu}$ können. Die Präparation erfolgt i.d.R. in einer Blutsperre/Blutleere. Im weiteren Verlauf muss ggf. eine erneute Anpassung des Behandlungsplans an die aktuelle Situation erfolgen. Das zu erreichende funktionelle Ergebnis sowie mögliche Komplikationen und Schwierigkeiten müssen regelmäßig, insbesondere nach Folgeoperationen, reevaluiert werden.

Übungsstabile Osteosynthesen mit dem Ziel einer frühfunktionellen Nachbehandlung gewinnen zunehmend an Bedeutung.

Diese sollten jedoch nicht zu einer weiteren Traumatisierung der Weichteile führen. Weiterhin ist hier die weichteilschonende Kirschner-Draht-Versorgung oder die Anlage eines Fixateur externe zur Überbrückung von Defektstrecken etabliert und im Zweifelsfall zu bevorzugen. Aufwendige knöcherne Rekonstruktionen, z.B. mit Beckenkammspann, werden meist sekundär durchgeführt. Bei Gelenkdestruktionen ist oft eine primäre Arthrodese des betroffenen Gelenks sinnvoll (Abb. 3).
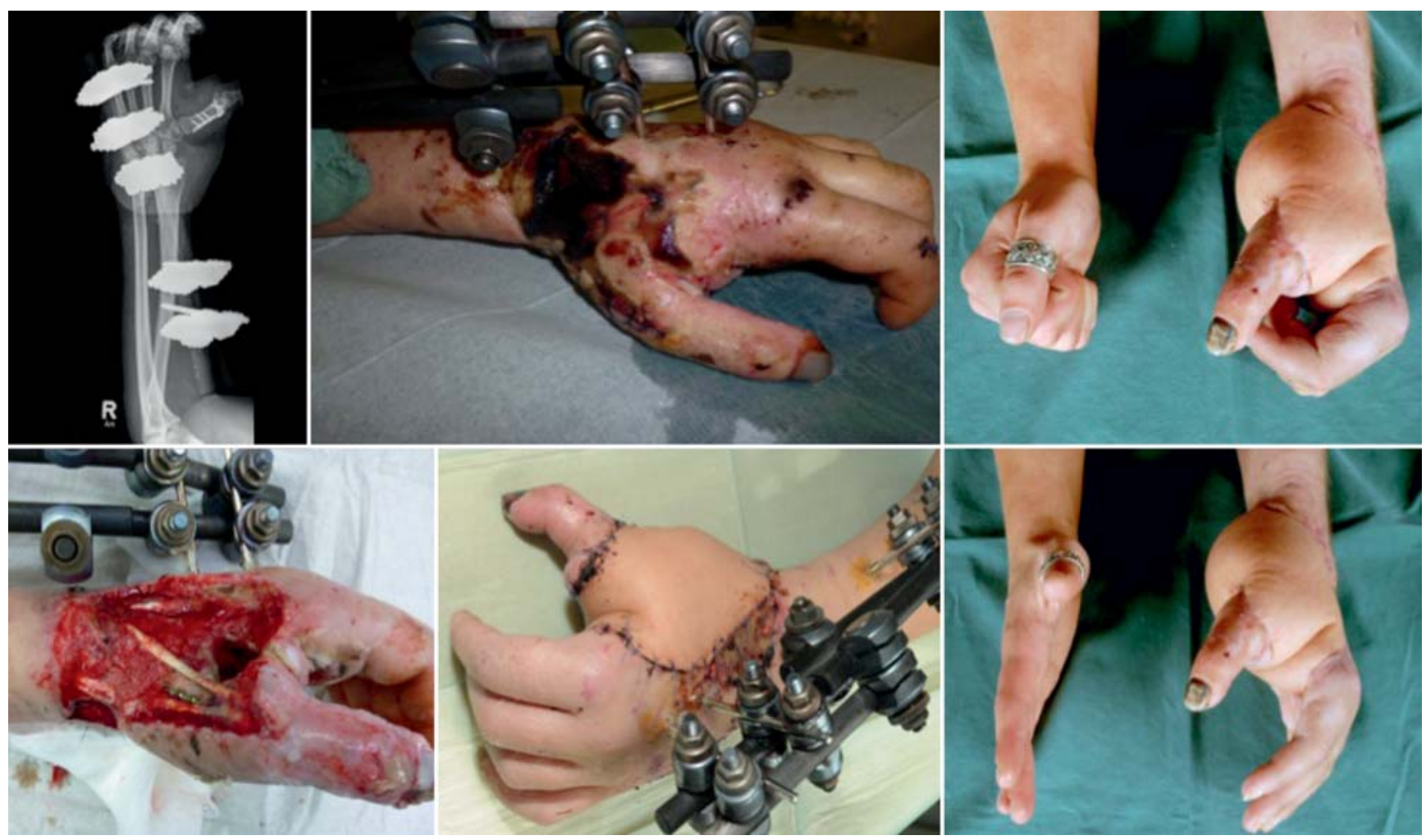

Abb. 3 32-jährige Patientin mit radiokarpaler Luxation und drittgradig offener MHK-I-Fraktur. Initial erfolgte die Stabilisierung des Handgelenks mittels Fixateur externe, eine Plattenosteosynthese am Mittelhandknochen, eine Patchplastik der A. ulnaris sowie eine Embolektomie und Thrombektomie des Arcus palmaris superficialis und profundus. Im weiteren Verlauf demarkierte sich ein ausgeprägter streck- und beugeseitiger Weichteildefekt mit Zerstörung der Thenar- und Hypothenarmuskulatur. Wir führten mehrfache Débridements mit temporärer Weichteildeckung mittels VAC-Verbands durch. Nach Konsolidierung der Haut-/Weichteile konnte eine suffiziente Weichteildeckung mittels freien Paraskarpularlappen geschaffen werden. Hierdurch wurde ein maximaler Längenerhalt des Daumens gesichert. 

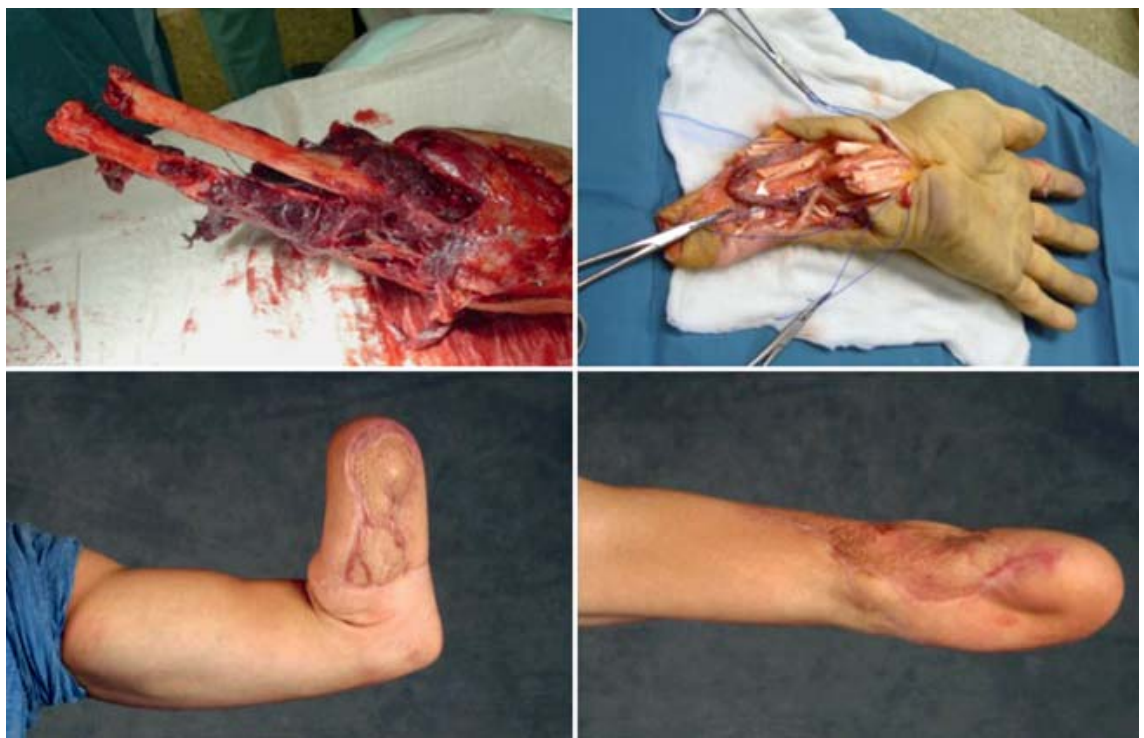

Abb.4a 47-jähriger Patient mit einer kompletten Unterarmamputation. Aufgrund des Zustands des Amputats erfolgte die Bildung eines Unterarmstumpfs mit mehrfachen Débridements und freier Latissimus-dorsi-Lappentransplantation zum maximalen Längenerhalt.
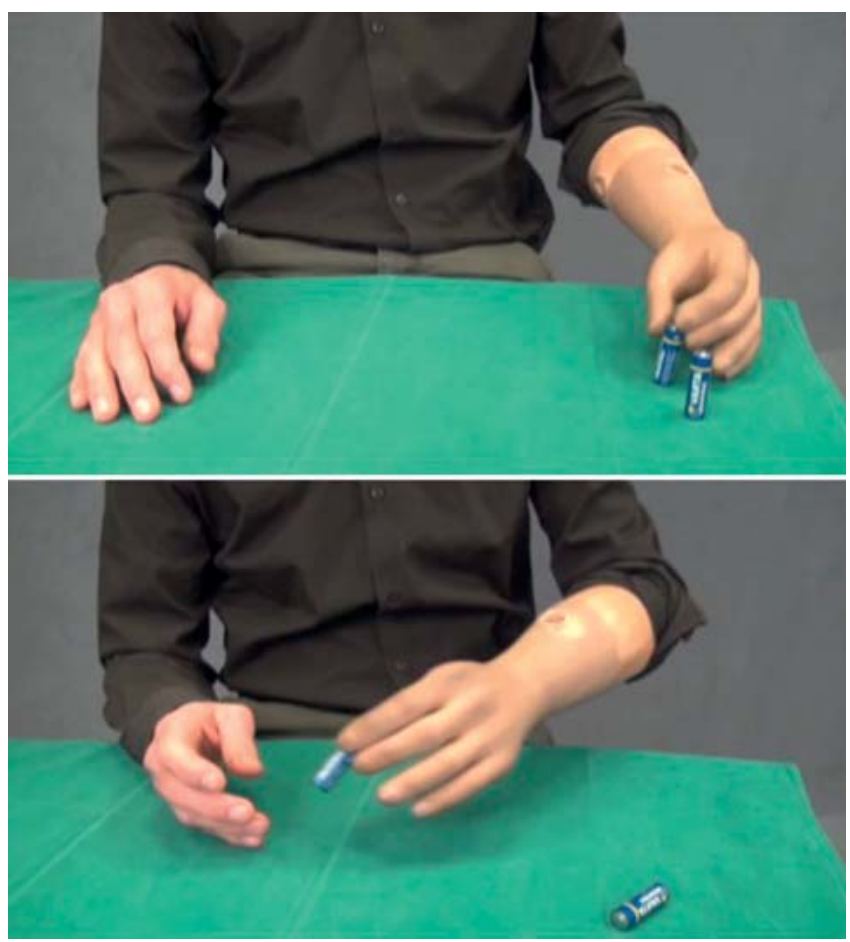

Abb. 4b Nach suffizienter Stumpfversorgung konnte eine myoelektrische Unterarmprothese angepasst werden. Über Elektroden, die eine Muskelaktivität im Stumpf erfassen, werden elektrische Motoren angesteuert, die Greif- und Umwendbewegungen der Hand ermöglichen.

Zudem sollte intraoperativ der Ausschluss von ligamentären Instabilitäten oder Luxationen durch Bildwandlerkontrolle erfolgen.

Im Anschluss sollte die Durchblutung erneut geprüft werden und ggf. eine Revaskularisierung erfolgen. Für langstreckige Defekte eignet sich die V. saphena magna zur Interposition. Alternativ können auch Venen vom Unterarm entnommen werden.
Abhängig vom Zugang und der Art der Verletzung sollte die Sehnennaht vor oder nach der Revaskularsierung erfolgen. Hierbei gilt es nach Möglichkeit, eine primäre Sehnennaht durchzuführen.

Die oberflächlichen Beugesehnen müssen bei guter Funktion oder Naht der tiefen Beugesehnen nicht zwangsläufig versorgt werden.

Bei komplexen Verletzungen oder langstreckigen Defekten ist meist eine sekun- däre Rekonstruktion sinnvoll (Abb. 3). Zwar hat die Sensibilität für die Funktionalität eine entscheidende Bedeutung, jedoch gilt es, diese als letzten Punkt bei der Rekonstruktion im akuten Stadium durchzuführen. Nur intakte Nervenstümpfe sollten spannungsfrei koaptiert werden. Bei Nervendefekten $<1,5 \mathrm{~cm}$ kann in der Akutsituation eine Rekonstruktion mit Einlage eines Nerve Tubes versucht werden.

Einige Autoren empfehlen eine primäre Rekonstruktion mittels Venen-MuskelInterponats bei Defekten bis $6 \mathrm{~cm}$.

Dabei zeigt sich in Studien kein wesentlicher Unterschied im Vergleich zur Nerveninterposition und es ergibt sich der Vorteil, Nerventransplantate für evtl. spätere Rekonstruktion aufheben zu können. Bei Verletzungen mit langstreckigem Nervendefekt empfiehlt sich eine Nerventransplantation frühsekundär nach 3-6 Monaten.

Zudem sollte bei stark geschwollener Extremität die Indikation zur Spaltung des Retinaculum flexorum zur Vermeidung sekundärer neurologischer Schäden großzügig gestellt werden.

In der Regel erfolgt ein temporärer HautWeichteil-Verschluss mittels Epigard oder Vakuumversiegelung, sollte eine spannungsfreie Primärnaht nicht möglich sein. Im weiteren Verlauf sollte dann nach Konsolidierung eine adäquate Weichteildeckung mittels lokaler Lappenplastiken, gestielten Fernlappen oder auch durch mikrochirurgische Gewebetransplantationen erfolgen.

Nur durch eine intensive postoperative Nachbehandlung kann das geschaffene Potenzial durch die Rekonstruktion der komplexen Handverletzung ausgenutzt werden.

Hierzu gehört initial eine konsequente Hochlagerung, Lymphdrainage, intensive krankengymnastische sowie ergotherapeutische frühfunktionelle Beübungstherapie sowie in den meisten Fällen im weiteren Verlauf nach abgeschlossener Wundheilung und Konsolidierung der Fraktur eine Rehabilitation in einem geeigneten handchirurgisch versierten Rehabilitationszentrum, z. B. im Sinne einer komplexen stationären Rehabilitation (KSR). Hier sollte der Bewegungsaufbau und die Kraftausdauer verbessert werden mit dem Ziel der Wiedererlangung der Handfunktion sowie der Wiederein- 
gliederung in das Berufsleben (Abb.4a und $\mathbf{b}$ ).

Auch an die Notwendigkeit einer psychologischen Mitbehandlung und sozialen Unterstützung muss nach einer komplexen Handverletzung immer gedacht werden.

Dies sollte bereits im Rahmen der stationären oder weiteren ambulanten Betreuung initiiert werden.
Prof. Dr. med. Marcus Lehnhardt

Direktor der Klinik für Plastische Chirurgie und Schwerbrandverletzte, Handchirurgiezentrum, Operatives Referenzzentrum für Gliedmaßentumoren

Prof. Dr. med. Adrien Daigeler

Leitender Oberarzt

Facharzt für Plastische und Ästhetische Chirurgie Zusatzbezeichnungen Handchirurgie, Intensivmedizin, Medizindidaktik
Priv.-Doz. Dr. med. Andrej Ring Oberarzt der Klinik Facharzt für Chirurgie, Facharzt für Plastische und Ästhetische Chirurgie, Zusatzbezeichnung Handchirurgie Dr. med. Amir Farhang Gharagozlou Assistenzarzt

Klinik für Plastische Chirurgie und Schwerbrandverletzte, Handchirurgiezentrum, Operatives Referenzzentrum für Gliedmaßentumoren

Berufsgenossenschaftliches Universitätsklinikum Bergmannsheil Bürkle-de-la-Camp-Platz 1 44789 Bochum

farhang.gharagozlou@ bergmannsheil.de 